\title{
Contrast improvement by using tailored laser pulses to circumvent undesired excitations
}

I. Kumberg

A. Kussicke

A. Patas

A. Lindinger

(c) (1) $\Theta \Theta$

Institut für Experimentalphysik, Freie Universität Berlin, Arnimallee 14, D-14195 Berlin, Germany

Abstract

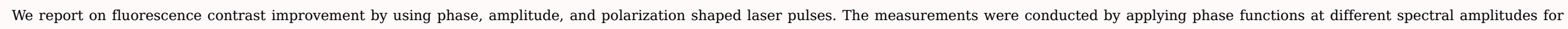

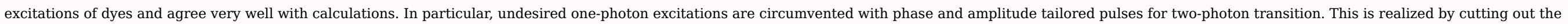

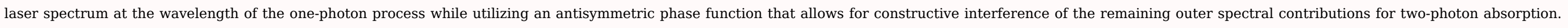

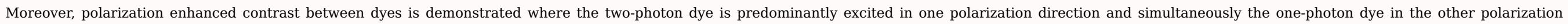
direction. The presented methods of shaping ultrashort laser pulses have a high potential for imaging applications.

MSC: 00-01; 99-00

Keywords: Ultrashort laser pulses; Laser pulse shaping; Multiphoton excitation

\section{Introduction}

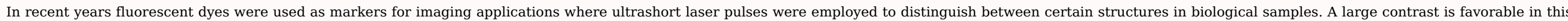

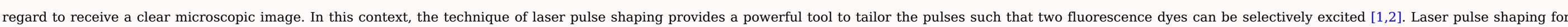

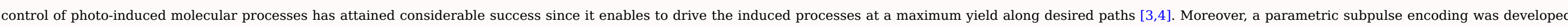

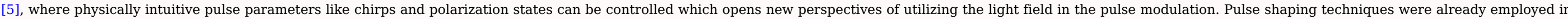

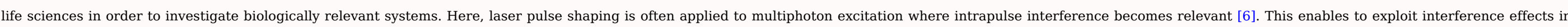

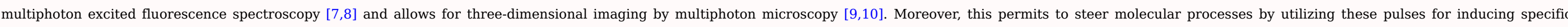

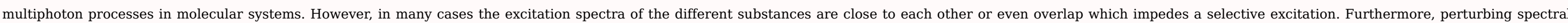
features may hamper the intended excitations of the examined species. Methods that allow to bypass these unwanted transitions would be desirable in order to improve the received contrast.

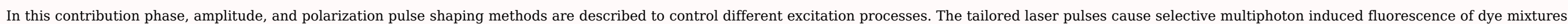

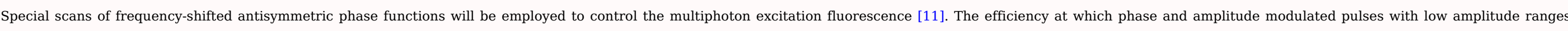

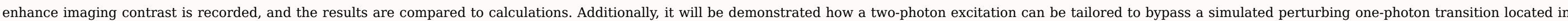

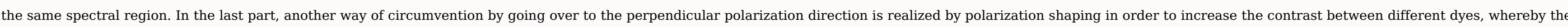

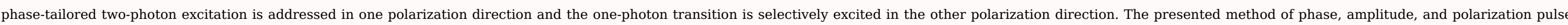




\section{Experimental setup}

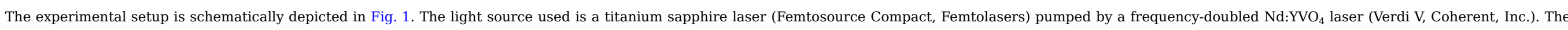

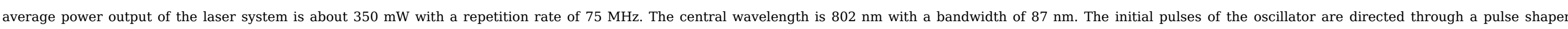

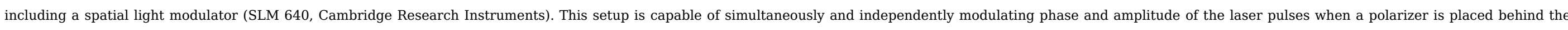

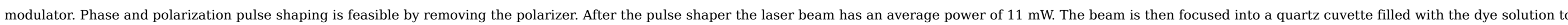

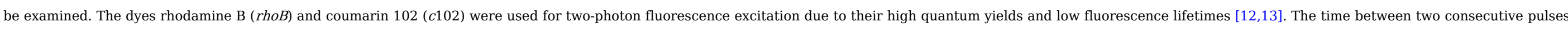

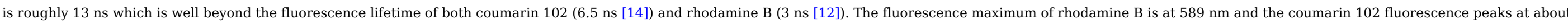

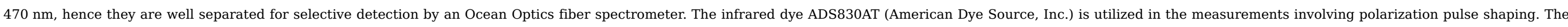
dyes were solvated in ethanol for the primary measurements, and in glycerol for the polarization shaping experiments.

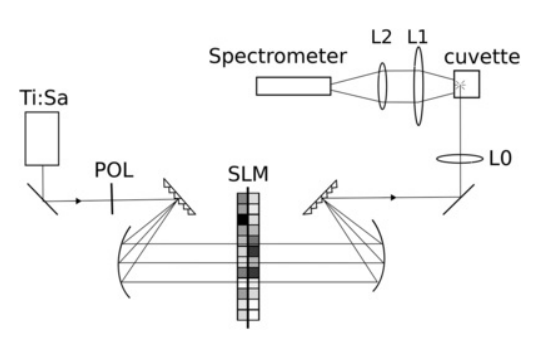

Fig. 1 Experimental setup where the laser (Ti:Sa), the spatial light modulator (SLM), the lenses (L0,L1,L2), the quartz cuvette, and the spectrometer are depicted schematically.

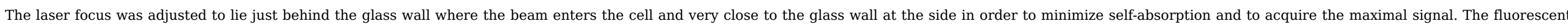

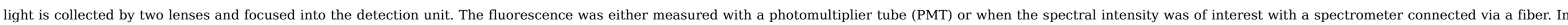

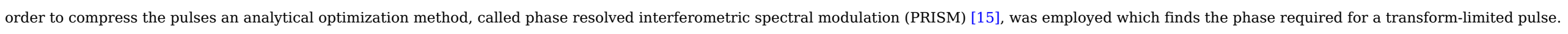

\section{Results}

\subsection{Phase scans with amplitude shaped pulses employed for two-photon excitation of different dyes}

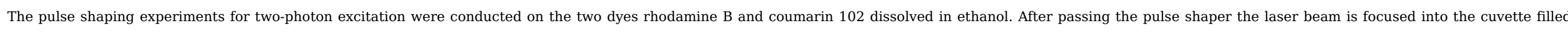

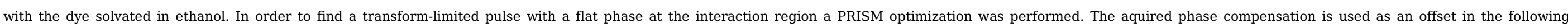

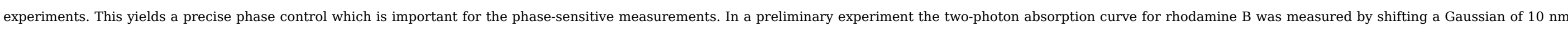

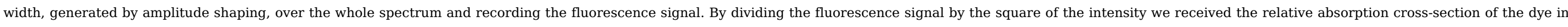
the accessible wavelength range. For coumarin 102 the two-photon absorption curve measured in [16] was employed for calculations.

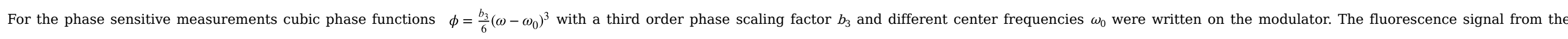

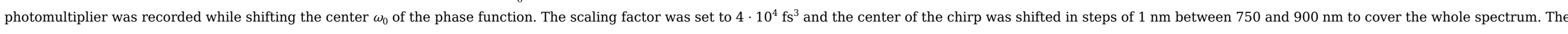

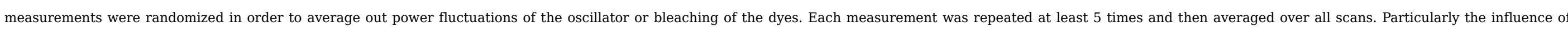

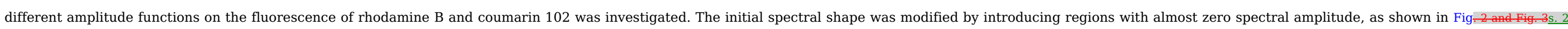
and 3. The intensity in these regions did not go down to zero but to below $6 \%$ due to some light leaking through the shaper that is not absorbed by the polarizer. 

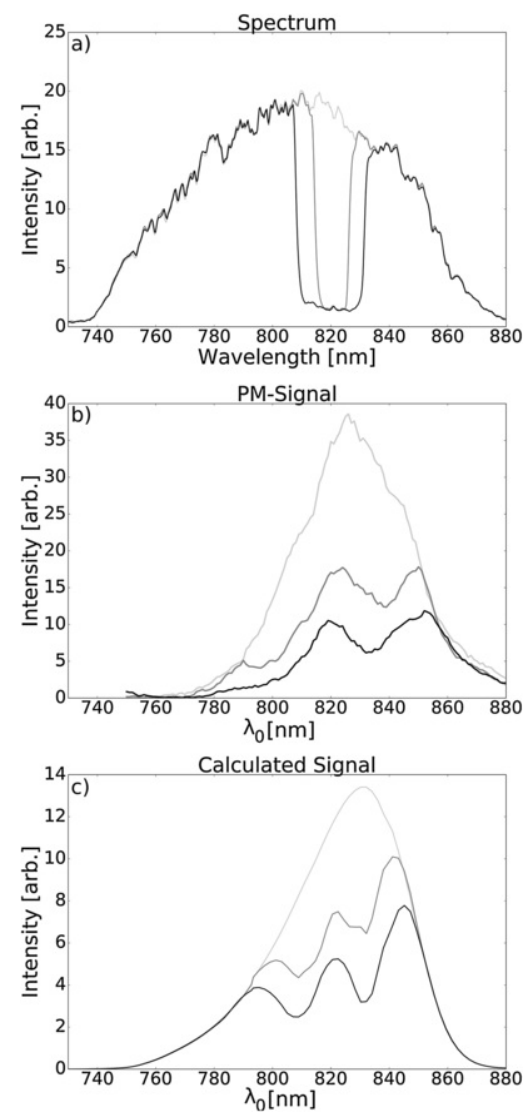

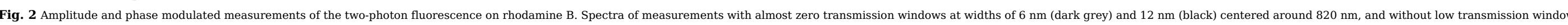
(light grey), are depicted in (a). The measured phase scan fluorescence signals with different window sizes of almost zero transmission are depicted in (b) for a scaling factor of $b_{3}=4 \cdot 10^{4} \mathrm{fs}^{3}$. The calculated two-photon signals are plotted in (c). 

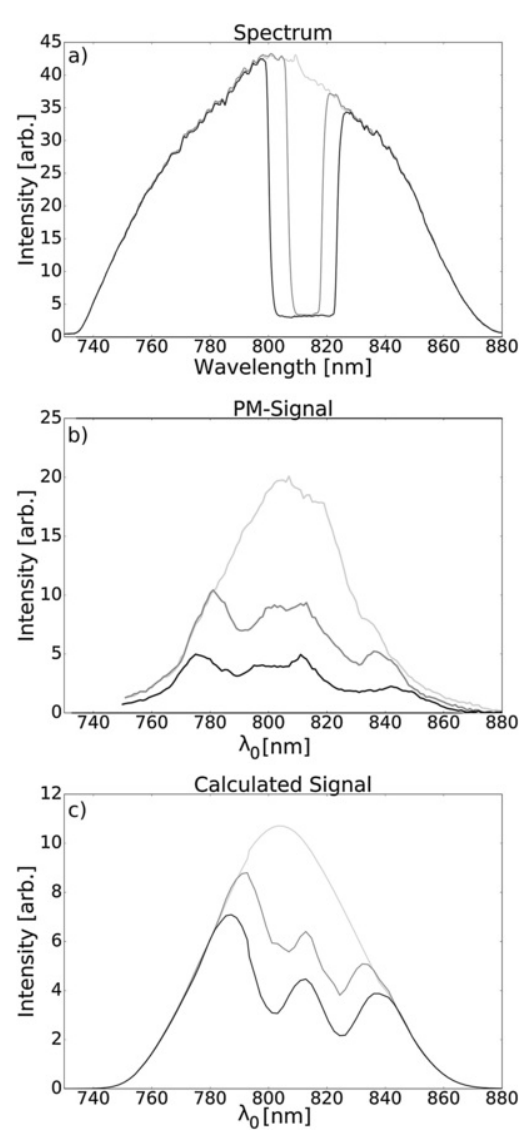

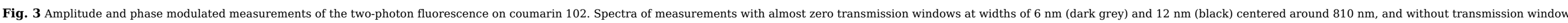
(light grey), are shown in (a). The measured phase scan fluorescence signals with different window sizes of transmission zero are depicted in (b) for a scaling factor of $4 \cdot 10^{4} \mathrm{fs}^{3}$. The calculated two-photon signals are plotted in (c).

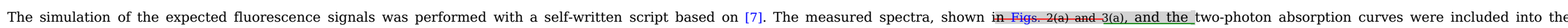

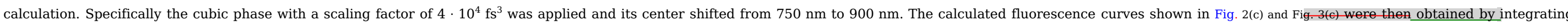
over the two-photon absorption.

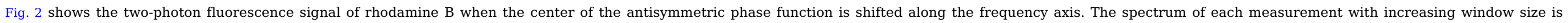

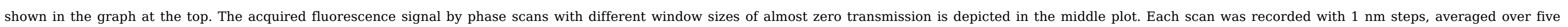

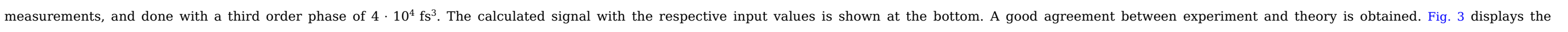
corresponding results for coumarin 102 including calculations.

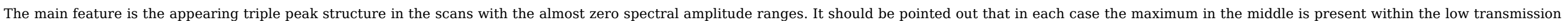

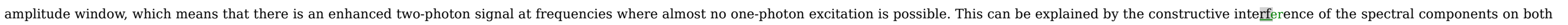

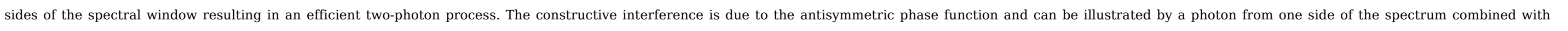
another photon from the other side of the spectrum arriving at the same time.

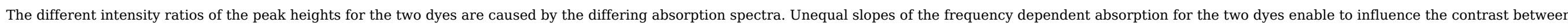




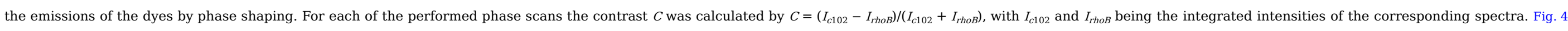

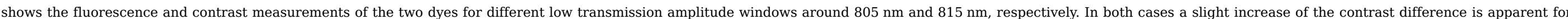
the measurements with amplitude windows.
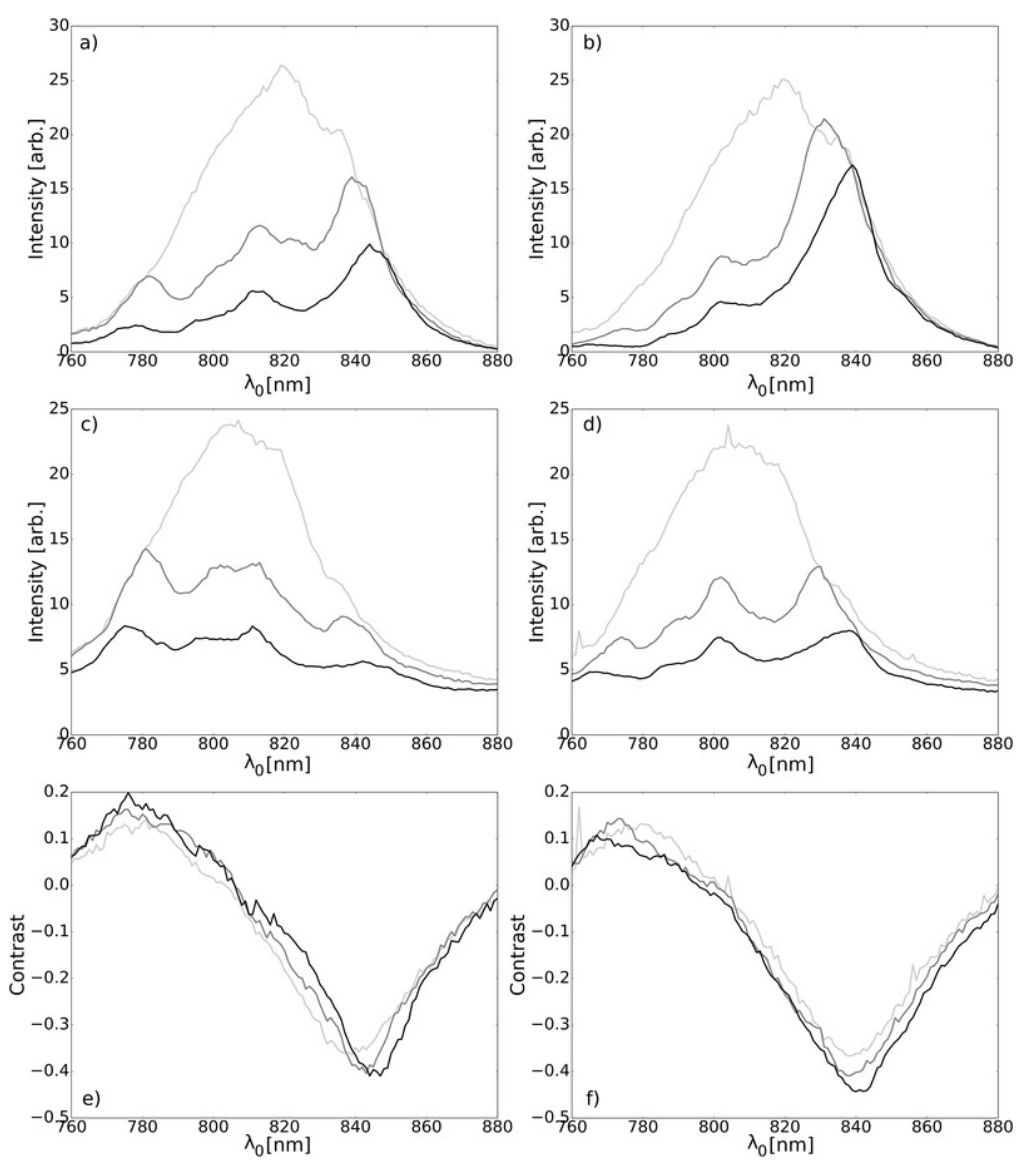

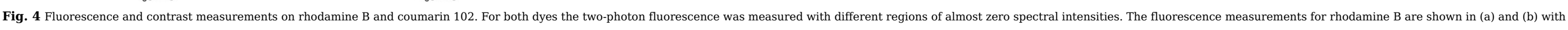

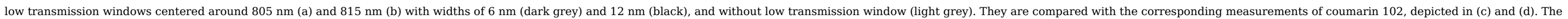
contrast between the measurements is shown in (e) with low transmission windows around $805 \mathrm{~nm}$ and in (f) with windows around $815 \mathrm{~nm}$.

\subsection{Circumvention by two-photon excitation}

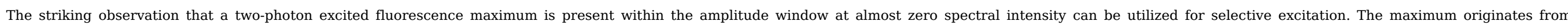

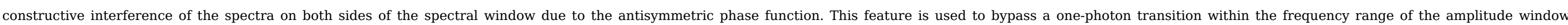

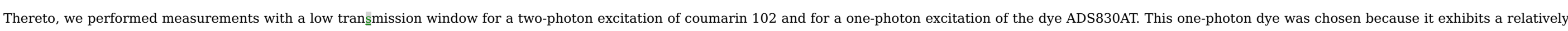
narrow absorption maximum close to the window position.

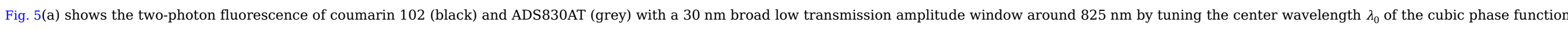

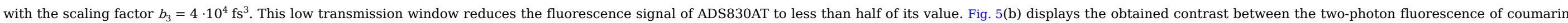



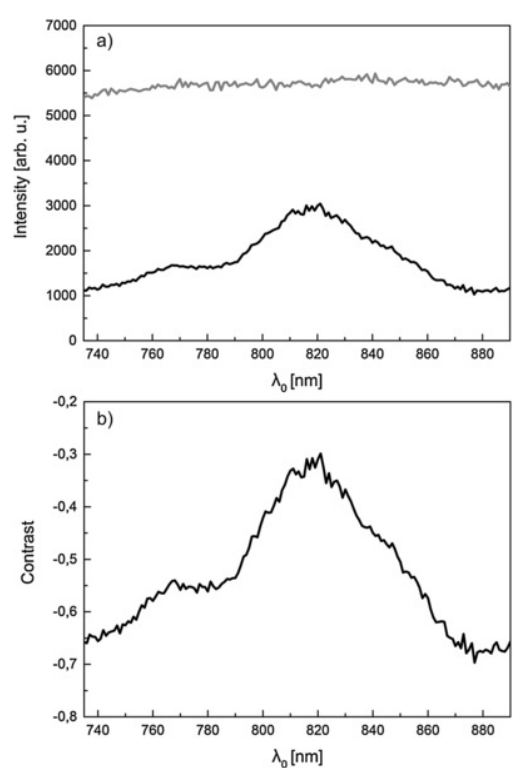

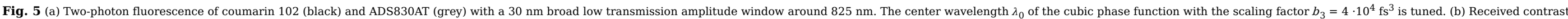

between the two-photon fluorescence of coumarin 102 and ADS830AT with the amplitude window. An increased contrast is obtained at the window position.

\subsection{Contrast improvement by polarization shaping}

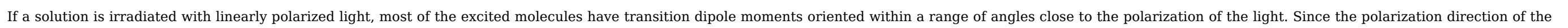

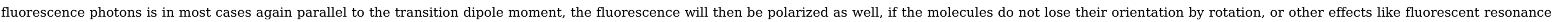

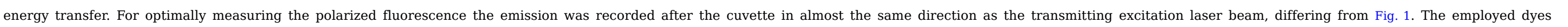

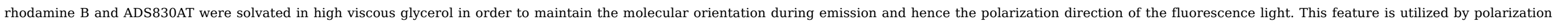

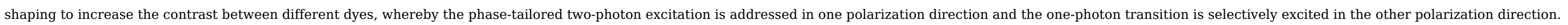

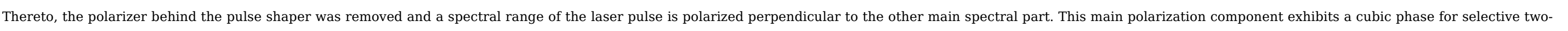

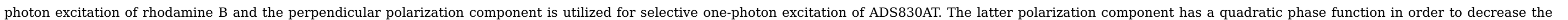
unwanted two-photon excitation of rhodamine B.

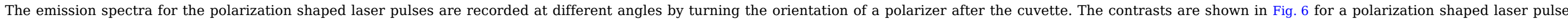

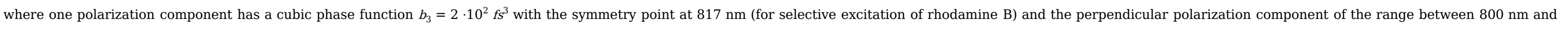

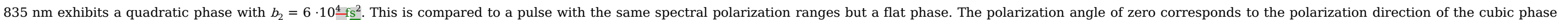

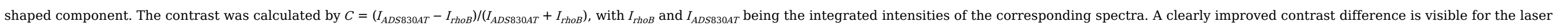
pulse with the phase-shaped polarization components. 


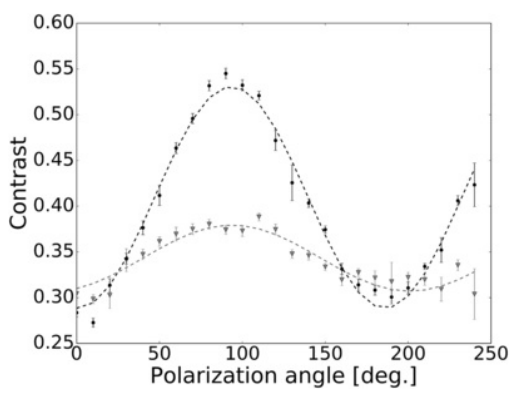

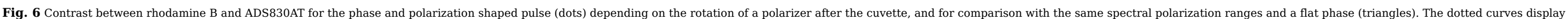
sinusoidal fits of the data. An enhanced contrast difference is obtained for the laser pulse with the phase-shaped polarization components.

\section{Conclusions}

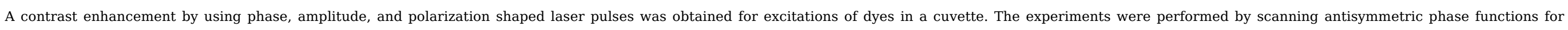

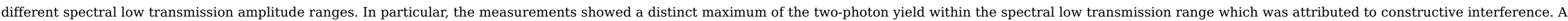

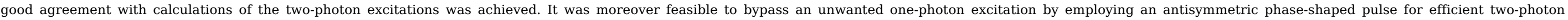

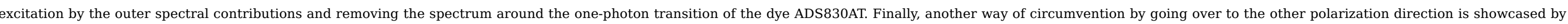

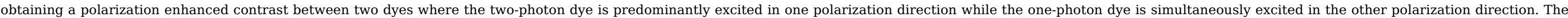
described pulse shaping methods for selective photoexcitation reveal novel perspectives for biophotonic applications.

\section{Acknowledgment}

The authors thank Prof. Dr. Wöste for his encouragement and the Klaus Tschira Foundation (KTS) is acknowledged for financial support (00.314.2017).

\section{References}

[1] T. Brixner, N.H. Damrauer, P. Niklaus and G. Gerber, Photoselective adaptive femtosecond quantum control in the liquid phase, Nature 414, $2001,57-60$.

[2] M. Roth, L. Guyon, J. Roslund, V. Boutou, F. Courvoisier, J.-P. Wolf and H. Rabitz, Quantum control of tightly competitive product channels, Phys. Rev. Lett. 102, 2009, 253001.

[3] R.S. Judson and H. Rabitz, Teaching lasers to control molecules, Phys. Rev. Lett. 68, 1992, 1500-1503.

[4] T. Brixner and G. Gerber, Quantum control of gas-phase and liquid-phase femtochemistry, ChemPhysChem 4, 2003, 418-438.

[5] F. Weise and A. Lindinger, Full parametric pulse shaping in phase, amplitude, and polarization using an effective four array modulator, Appl. Phys. B 101, 2010 , 79-91.

[6] D. Meshulach and Y. Silberberg, Coherent quantum control of two-photon transitions by a femtosecond laser pulse, Nature 396, 1998, $239-242$.

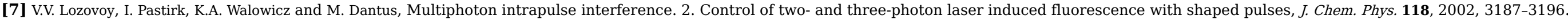

[8] D. Meshulach and Y. Silberberg, Coherent quantum control of multiphoton transitions by shaped ultrashort opical pulsea, Phys. Rev. A 60, 1999, 1287-1292.

[9] W. Denk, J.H. Strickler and W.W. Webb, Two-photon laser scanning fluorescence microscopy, Science 248, 1990, 73-76.

[10] S.W. Perry, R. Burke and E. Brown, Two-photon and second harmonic microscopy in clinical and translational cancer research, Ann. Biomed. Eng. 40, 2012 , 277-291. 


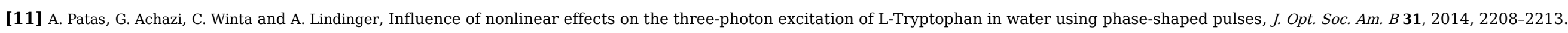

[12] M.J. Snare, F.E. Treloar, K.P. Ghiggino and P.J. Thistllethwaite, The photophysics of rhodamine B, J. Photochem. 18, 1982, $335-346$.

[13] R.F. Kubin and A.N. Fletcher, Fluorescence quantum yields of some rhodamine dyes, J. Luminescence 27, 1982, 455-462.

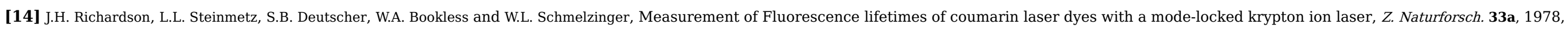
1592-1593.

[15] T. Wu, J. Tang, B. Hajj and M. Cui, Phase resolved interferometric spectral modulation (PRISM) for ultrafast pulse measurement and compression, Opt. Express 19, 2011, 12961-12968.

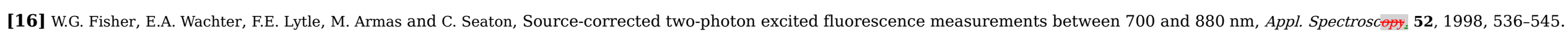

